\title{
Mapping mineral prospectivity via convolutional neural network
}

\author{
TONG LI ${ }^{1}$ RENGUANG ZUO ${ }^{2}$
}

${ }^{1}$ China University of Geosciences (Wuhan).No.78 Jincheng

Street, Wuhan, China. E-mail:litong@cug.edu.cn

${ }^{2}$ China University of Geosciences (Wuhan).No.78 Jincheng

Street, Wuhan, China. E-mail:zrguang@cug.edu.cn

Convolutional Neural Network (CNN) has been proven to be an effective tool for classification and prediction. In this contribution, $\mathrm{CNN}$ is employed for mineral prospectivity mapping (MPM) in the southwestern Fujian Province, China. Two issues limit the development of CNN in MPM. One is the high-dimension and complex spatial pattern of geoscience data; the other is the scarcity of labeled samples (known deposits) for training a high-capacity $\mathrm{CNN}$ model. To address these two issues, a data augmentation method which took randomly drop-outs from the data repetitively was firstly proposed to obtain enough samples for training, and then a modified CNN architecture for MPM. A variety of hyperparameters like the size and the amount of kernels of each convolutional layer, the location of the max-polling layers, the size of the fully connected layers, learning rates and iterations were investigated to obtained the optimal hyperparameters of $\mathrm{CNN}$. This well-trained $\mathrm{CNN}$ model was applied on a testing area which is completely unknown for the network. The mapped areas show a strong spatial relationship with the known mineral deposits, most of the known $\mathrm{Fe}$ deposits are located in the areas with high probability $(>0.99)$. The findings indicate that (1) this random-drop data augmentation method is suitable and effective for supervised machine learning methods, and (2) such modified CNN is a potential technique for integration of multi-source geoscience data and a feasible method to guide further exploration.

Key words: mineral prospectivity mapping, machine learning, $\mathrm{CNN}$, data augmentation. 